\title{
Captación De Potenciales Eléctricos Oculares Para Su Uso Viable En Interfaz Hombre-Máquina
}

\author{
Milky Rodriguez, \\ Suhey García, \\ Jay Molino,
}

Departmento de Biociencias y Salud Pública, Corregimiento de Ancón, Albrook, Paseo Diógenes de la Rosa, Panamá, Universidad Especializada de las Américas A quien la correspondencia debe ser dirigida

Doi:10.19044/esj.2020.v16n18p102 URL:http://dx.doi.org/10.19044/esj.2020.v16n18p102

\section{Resumen}

La captación de señales oculares es útil para el control en interfaz hombre-máquina (IHM) en pacientes que sufren de enfermedades motoras incapacitantes (e.g. Esclerosis lateral amiotrófica, o infortunados eventos que dejan al paciente con una severa discapacidad como un derrame cerebral o accidentes vehiculares) de manera tal que puedan usar el movimiento ocular como sistema de control en interfaz hombre-máquina para el mejoramiento de la calidad de vida de estos pacientes. Para ello, esta investigación tiene por objetivo desarrollar un dispositivo de bajo costo para la captación de señales oculares que permita ser empleado en IHMs. Materiales y métodos: Se diseñó un dispositivo que fue validado en una población de diez estudiantes adultos de la Facultad de Ingeniería Biomédica de la Universidad Especializada de las Américas (Panamá) y se registraron los potenciales del movimiento ocular vertical y horizontal. Resultados: Se confirma que la magnitud de la señal es directamente proporcional al desplazamiento ocular y que no hay correlación estadística significativa entre los potenciales para una distancia de desplazamiento ocular específica, es decir, tanto el ojo izquierdo como el derecho registraron el mismo potencial para las mismas distancias. El potencial mínimo registrado fue de $88,0 \pm 16,8 \mathrm{mV}$ y el máximo de $382,0 \pm$ $72,08 \mathrm{mV}$. El dispositivo se vinculó a una bombilla de intensidad lumínica variable de manera tal que, dependiendo del potencial registrado, la intensidad de la bombilla varía. Esto demuestra que el dispositivo puede ser usado como interfaz en una IHM. Conclusión: El análisis estadístico de la investigación brinda confiabilidad a los resultados captados por el dispositivo diseñado (captador de señales mioeléctricas). Al encender el led se muestra la posibilidad de que el dispositivo sea empleado para interfaz hombre-máquina. 
Palabras claves: Electrooculografía, Discapacidad, Interfaz HombreMáquina, Movimiento De Ojos, Interfaz Gráfica.

\title{
The Capture of Eye Electrical Potential for Viable Use in Human-Machine Interfaces
}

\author{
Milky Rodriguez, \\ Suhey García, \\ Jay Molino,
}

Departmento de Biociencias y Salud Pública, Corregimiento de Ancón, Albrook, Paseo Diógenes de la Rosa, Panamá, Universidad Especializada de las Américas A quien la correspondencia debe ser dirigida

\begin{abstract}
The capture of ocular signals is useful for controlling human-machine interfaces in patients suffering from disabling motor diseases (e.g., amyotrophic lateral sclerosis, or unfortunate events, such as accidents, that leave the patient with a severe disability). Such interfaces aim to improve their quality of life. Thus, this research aims to develop a low-cost device for capturing eye signals that allow it to be used in HMIs. Materials and methods: A device was designed and validated in a population of 10 adult students from the Faculty of Biomedical Engineering of the Specialized University of the Americas (Panama), and the potential of vertical and horizontal eye movement were recorded. Results: The magnitude of the signal is directly proportional to the ocular displacement. Furthermore, there is no significant statistical difference among the recorded potential for a specific ocular displacement; that is, both the left and the right eye marked the same potential for same displaced distances. The minimum potential recorded was $88.0 \pm 16.8 \mathrm{mV}$, and the maximum was $382.0 \pm 72.08 \mathrm{mV}$. The device was connected to a variable intensity light bulb, and depending on the registered potential, the intensity of the light changed. Thus, the device could be used as an interface in an HMI. Conclusion: The statistical analysis showed the reliability of the designed device (myoelectric signal collector). When the LED is switched on, the possibility of the equipment being used for humanmachine interfaces is shown.
\end{abstract}

Keywords: Electrooculography, Disabilities, Human Machine Interface, Eye Movement, Graphic Interface 


\section{Introducción}

En la última década se han desarrollado varias tecnologías asistidas para personas con discapacidades. Esto se debe a un gran interés por entender el comportamiento humano en áreas donde patrones puedan ser registrados para la ejecución de una actividad.

La creación de nuevos sensores de uso comercial para el reconocimiento de actividades, i.e. giroscopios, ritmo cardíaco, entre otros, han abierto camino para el desarrollo de más dispositivos móviles que miden otro tipo de actividades. Una de estas aplicaciones de reconocimiento de patrones para el desarrollo de IHM es el reconocimiento de voz para el manejo de equipos o la lecto-escritura (Jacob, 1993). Por supuesto, dado a que usamos nuestros ojos para todas las actividades que hacemos, el estudio del movimiento ocular también es de gran interés para IHM. Un ejemplo es la video-oculografía $\mathrm{u}$ oculografía infrarroja (OGI) para poder detectar expresiones, gestos y el movimiento de los ojos y con ellas realizar alguna actividad como mover un puntero de mouse. (Mahecha et al., 2008; Singh et. al. 2016)

El método más sencillo para medir el movimiento ocular se fundamenta en el hecho de que el ojo humano es un dipolo eléctrico. El eje de este dipolo y el eje del ojo humano son colineales y al ser la retina más negativa que la córnea existe una diferencia de $6 \mathrm{mV}$ como resultado de la actividad eléctrica de los fotorreceptores y las neuronas en la retina. El movimiento de los ojos causa una rotación del dipolo lo que resulta en una diferencia de potencial eléctrico que es perceptible en la piel de la persona. A este potencial se le conoce como electrooculografía. (Bulling et al. 2011; Wade et al. 2009; Wade et al. 2003).

En el marco de la ingeniería en rehabilitación, la electrooculografía (EOG) ha sido empleada como una herramienta para el desarrollo de módulos o sistemas de interacción entre un sujeto con un nivel de discapacidad física y su entorno cercano, mediante la medición de los potenciales eléctricos oculares generados por el movimiento de los ojos resultado de la hiperpolarización y despolarización de la retina. El potencial en el ojo puede ser estimado al medir el voltaje inducido a través de un sistema de electrodos ubicados en la periferia del ojo y estos cinco electrodos registran el electrooculografía. El paciente hacia al cual se orientan estas ayudas técnicas es el lesionado medular, típicamente personas cuadripléjicas o parapléjicas que han perdido la movilidad de los miembros superiores y no pueden manipular objetos o artefactos de su medio debido a que estos fueron diseñados inicialmente por y para usuarios sin discapacidad. Por ende, el diseño normal de los artefactos ignora las dificultades del uso que plantearía a los usuarios afectados por disfuncionalidades físicas y motoras. El desarrollo de interfaz hombre-máquina cuyo controlador es el potencial eléctrico ocular 
es de gran relevancia. (Atique et al., 2016; Dalgaard et al., 2007(a); Dalgaard et al., 2007 (b); Heide et al., 1999; Mahecha et al., 2008)

Para el desarrollo de instrumentos captadores de señales, se deben considerar las fuentes de interferencias ambientales y biológicas, por lo tanto, el diseño debe contemplar aspectos esenciales como amplificación, ancho de banda, impedancia de entrada, nivel bajo de ruido y estabilidad frente a las fluctuaciones de temperatura y el voltaje. Atención especial debe prestarse en el diseño del amplificador de instrumentación.

En Panamá, el área de motilidad ocular y electrofisiología de la visión no es investigada. A nivel físico se realizan diversos exámenes neurofisiológicos en clínicas y hospitales públicos y privados con propósitos de diagnósticos. Algunos de estos son estudios de la electrofisiología de la visión. Entre estos estudios figuran: electroencefalogramas, electromiagramas, electroinstamogramas, electroretinogramas, electroocleogramas, velocidad de conducción nerviosa de miembros superiores e inferiores, tiempo de conducción del nervio facial, respuesta $\mathrm{F}$ y reflejo $\mathrm{H}$, reflejo de parpadeo, prueba de estimulación repetitiva, potencial evocado auditivo (umbral y clicks), potencial evocado visual, potencial evocado del nervio trigémino, entrenamiento neuromuscular por biofeedbackelectromiofeedback, entre otros. (Bulling et al. 2008; Rodriguez, 2011; Salvucci et al., 2001; Zhan et al., 2016)

Fundamentado en lo previamente expresado y dado a que en Panamá no se han documentado investigaciones relacionadas a la captación de potenciales electrooculográficos, y considerando las incidencia de casos de parálisis reportados por el instituto de Nacional de Medicina Física y Rehabilitación de Panamá, se realiza la presente investigación para diseñar y fabricar un prototipo para distinguir con claridad los movimientos oculares (electroolografìa), validar el prototipo y emplear las señales como controlador para encender un foco led. Este último es la prueba de concepto para demostrar la potencialidad del dispositivo como IHM. Las contribuciones específicas que hace la investigación son:

1. Desarrollar un dispositivo económicamente asequible para captar potenciales oculares en el escenario panameño.

2. Validar y corroborar la confiabilidad del equipo en la población panameña.

\section{Marco Metodológico}

a. Materiales:

Se emplearon los siguientes equipos: electrodos de electroencefalograma (EEG) de copa de plata de $6 \mathrm{~mm}$, osciloscopio fluke biomedical 199 x-ray medical scopemeter®, una fuente de poder de +12 voltios y -12voltios, una cinta métrica para medición de la cabeza, mampara 
para colocación de rostro, lámina de campimetría para la exploración de la motilidad ocular, algodón para limpieza de rostro y tape para fijación de electrodos.

Los siguientes reactivos o sustancias fueron utilizadas: Crema para EEG Y ECG fabricada por General Electric, y alcohol al 70\%. Todos los materiales tuvieron un costo menor a 50 dólares.

\section{b. Población y muestra:}

La población universa considerada para la realización del estudio corresponde a los miembros de la comunidad de Ingeniería Biomédica, la que también incluye a algunos profesores y tiene un total de 125 personas. Para la selección de la muestra se aplicó un instrumento con un cuestionario sobre la historia clínica de cada una de las personas para confirmar que no padecieran de enfermedades oculares o hubiera antecedentes familiares. De este cuestionario se reduce el grupo a quince personas a quienes se les aplicó un estudio de motilidad ocular con optómetras para corroborar los datos recogidos en la encuesta. El estudio consistió en pruebas de ducciones, versiones, ángulo kappa, test de Hischberg, punto próximo de convergencia y cover test (Martín et al., 2006; Wade et al., 2009; Wick et al., 1980). A partir de los resultados obtenidos la muestra se redujo a diez) personas que fueron empleadas para el estudio final. Previo a la medición de potenciales se realizan mediciones generales de la circunferencia, longitud del cantus externo izquierdo al cantus derecho externo, y la longitud de ceja a pupila. Seguidamente se prosigue a colocar los electrodos en las posiciones indicadas en la figura 1A. El paciente se ubica en una silla de altura ajustable mirando a una imagen que se encuentra a $50 \mathrm{~cm}$ en la cual se marcan puntos en el eje horizontal y vertical a 10, 20, 30, 40 y $50 \mathrm{~cm}$ del centro (Figura 1B). Así se registran las variaciones de potencial por movimiento ocular (malla de Amsler). Las zonas donde se coloca la silla es ajustable para asegurar que la posición basal de los ojos se ubique en el centro (Jager et al., 2008).
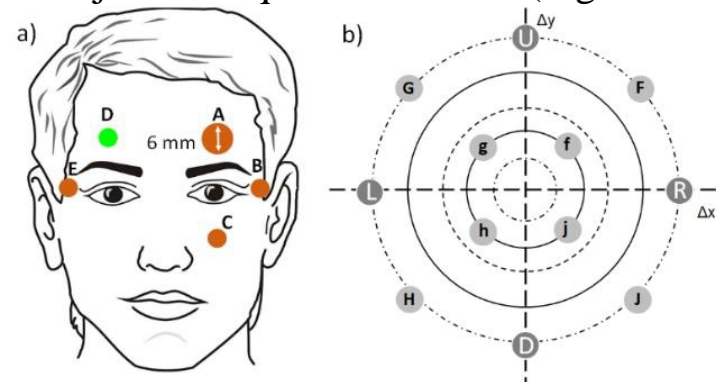

Figura 1: a. Posición de los electrodos $(\mathrm{A}=1$ pulgada de pupila hacia arriba, $\mathrm{B}=$ en la sien derecha del paciente, $\mathrm{C}=1$ pulgada de pupila hacia abajo, $\mathrm{D}=1$ pulgada de pupila hacia arriba, $\mathrm{E}=$ en la sien izquierda del paciente; $\mathrm{b}$. Ejes donde se registraron los movimientos de los ojos $\overline{R L}, \overline{F f}, \overline{U f}, \overline{G g}, \overline{H h}, \overline{J J}$ (cada círculo concéntrico tiene una diferencia en el radio de $10 \mathrm{~cm}$; U, R, D, L significa arriba, derecha, abajo, y arriba respectivamente). 


\section{c. Dispositivo}

El dispositivo diseñado consiste en unos electrodos de copa de plata que se conectan a diferentes puntos del rostro (figura 1a) usando un gel como medio conductor eléctrico entre ambas superficies (piel-electrodo) por ser de mayor conductividad eléctrica. Cada electrodo de plata permite el paso de las señales (mili-voltios) a través de la etapa de circuitería electrónica que será recibida por un acople de resistencias para ajustar el acople al paciente en función de la impedancia.

Una vez la señal captada es filtrada, y procesada/amplificada por un circuito de instrumentación, nuevamente la señal viaja por un filtro pasa banda que se compone de un filtro pasa bajo y pasa alto que a su vez también manejan la función de amplificar la señal (además de eliminar todo tipo de ruido existente dentro de su rango de trabajo). Este último filtrado de banda tiene una frecuencia de corte de para la señal del circuito de $0.5-50 \mathrm{~Hz}$. Esta última sección en el tratamiento de la señal permite emplear la señal con algún dispositivo convertidor analógico digital, o alguna plataforma de programación para desarrollar una aplicación para asistir, por ejemplo, a un paciente parapléjico, o incluso alguien quien haya salido de una cirugía que tenga una parálisis corporal temporal. Este circuito es alimentado por una fuente dual para mejorar la amplitud de rango de la señal. La amplificación de la señal fue de $10^{-6}$ voltios (input) a $10^{-3}$ voltios (output) usando un amplificador diferencial de alta. Esto se aprecia en la figura 2.

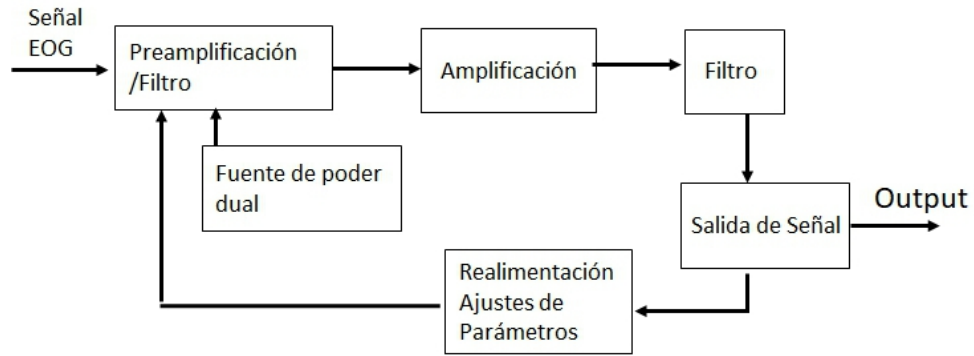

Figura 2: Esquemático del dispositivo empleado para la recolección y tratamiento de la señal.

Las señales de EOG pueden estar afectadas por la inclusión de ruido procedente de varios lugares (e.g. cableados de energía eléctrica, los equipos con el que se hacen las mediciones, los cables del circuito, el electrodo, o inclusive señales electromiografías). Adicional a esto, la actividad física continua puede causar que los electrodos no hagan buen contacto con la piel, convirtiéndose en una de las razones por las que las señales de EOG medidas no era repetibles. Esto es un reto, pues no se puede usar técnicas de eliminación de ruido basado en el conocimiento de la estructura y características de la señal. La figura 3 muestra el circuito de filtrado. 


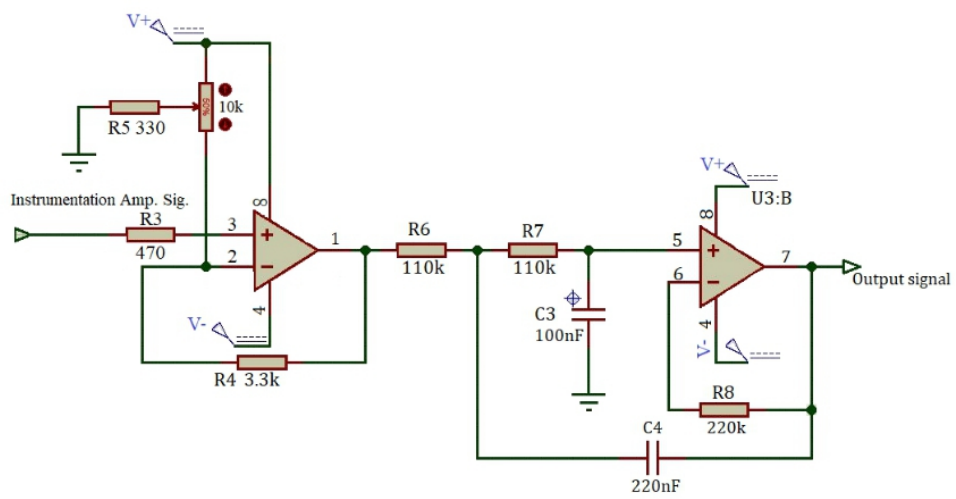

Figura 3: Esquemático de la fase de filtración empleada para el tratamiento de la señal.

Finalmente, el equipo se conecta a un LED que es representativo de un circuito módulo o cualquier otro dispositivo (máquina) que pueda activarse a través de una señal mioeléctrica ocular. Es decir, se demuestra el potencial que posee para ser implementado como interfaz hombre-máquina.

\section{Resultados y Discusión}

El potencial se midió para movimientos oculares horizontales y verticales como se establece en la sección de procedimiento. Los resultados de las mediciones fueron resumidos en la tabla 1.

Tabla 1: Potenciales eléctricos registrados en exploración horizontal y vertical

\begin{tabular}{l|lllll|lllll}
\hline $\begin{array}{l}\text { Pacient } \\
\mathrm{e}\end{array}$ & \multicolumn{7}{|l|}{ Exploración horizontal derecha $[\mathrm{mV}]$} & \multicolumn{2}{l}{ Exploración } \\
{$[\mathrm{mV}]$}
\end{tabular}




\begin{tabular}{l|lllll|lllll} 
P1 & 80 & 180 & 270 & 330 & 380 & 80 & 160 & 200 & 280 & 340 \\
P2 & 80 & 140 & 200 & 240 & 280 & 120 & 180 & 220 & 260 & 320 \\
P3 & 100 & 160 & 200 & 240 & 300 & 100 & 220 & 340 & 460 & 500 \\
P4 & 80 & 120 & 220 & 260 & 300 & 80 & 120 & 200 & 260 & 320 \\
P5 & 80 & 160 & 200 & 280 & 340 & 80 & 120 & 240 & 400 & 460 \\
P6 & 60 & 140 & 180 & 260 & 320 & 80 & 160 & 220 & 260 & 340 \\
P7 & 80 & 140 & 180 & 280 & 380 & 140 & 200 & 280 & 380 & 480 \\
P8 & 80 & 180 & 260 & 360 & 420 & 80 & 120 & 200 & 260 & 340 \\
P9 & 80 & 140 & 200 & 260 & 300 & 80 & 140 & 200 & 260 & 320 \\
P10 & 80 & 160 & 240 & 320 & 400 & 80 & 160 & 240 & 320 & 400 \\
\hline Media & 88 & 166 & 236 & 302 & 364 & 88 & 162 & 236 & 294 & 354 \\
Dev. & 16 & 38 & 32 & 43 & 54 & 18 & 34 & 43 & 47 & 51 \\
Std. & & & & & & & & & & \\
\hline
\end{tabular}

Se encontró que la magnitud del potencial es directamente proporcional a la distancia (ángulo de giro del ojo). Adicional a esto, el potencial registrado en el eje horizontal es independiente de la lateralidad, así como también lo es el EOG en la vertical. Esto se puede apreciar en la figura 4. Cabe señalar que la deviación estándar aumenta con la distancia.
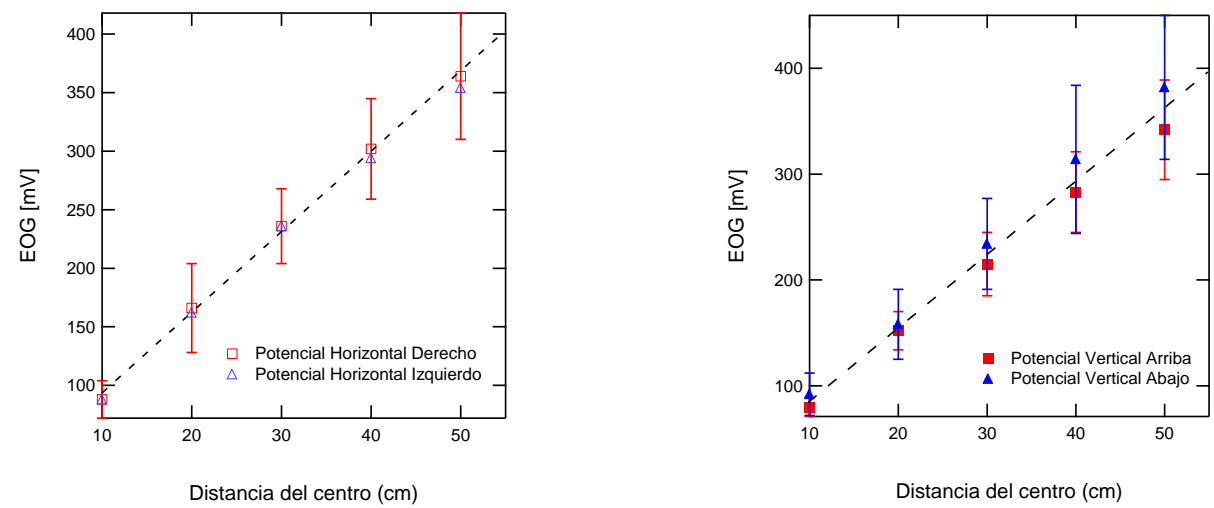

Figura 4: Medias y deviaciones estándar de

EOG para movimientos horizontales y verticales.

Se aplicó una prueba $\mathrm{T}$ de pares para determinar si no había correlación estadística significativa entre los puntos asumiendo que la distribución es normal. De esta forma se establece:

Ho: no hay correlación significativa entre las medias de los sets de medidas. Es decir, que los potenciales medidos a la misma distancia en el mismo eje (i.e. horizontal, $x$ ) son los mismos (e.g. $\mu_{1}=\mu_{2}$ ).

H1: Hay una correlación significativa entre ambas medias (e.g. $\mu_{1} \neq \mu_{2}$ ).

La manera en que se hicieron los pares de comparación se presenta en la tabla 2. 
Tabla 2: Prueba T por parejas

a).

\begin{tabular}{|c|c|c|c|c|}
\hline & & $\mathrm{N}$ & Correlación & Significancia \\
\hline $\begin{array}{l}\text { Potencial } \\
\text { Horizonta }\end{array}$ & $\begin{array}{l}\text { Horizontal } \\
\text { 1 Derecho }\end{array}$ & 10 & 0.264 & 0.460 \\
\hline $\begin{array}{l}\text { Potencial } \\
\text { Arriba }\end{array}$ & Horizontal Izquierdo-Vertical & 10 & 0.093 & 0.799 \\
\hline $\begin{array}{l}\text { Potencial } \\
\text { Abajo }\end{array}$ & Horizontal Izquierdo-Vertical & 10 & -0.146 & 0.687 \\
\hline $\begin{array}{l}\text { Potencial } \\
\text { Arriba }\end{array}$ & Horizontal Derecho-Vertical & 10 & -0.492 & 0.148 \\
\hline $\begin{array}{l}\text { Potencial } \\
\text { Abajo }\end{array}$ & Horizontal Derecho-Vertical & 10 & 0.703 & 0.23 \\
\hline
\end{tabular}

b).

\begin{tabular}{|c|c|c|c|c|c|c|c|c|}
\hline \multirow{3}{*}{ Par } & \multicolumn{5}{|c|}{ Diferencia de pares } & \multirow{3}{*}{$\mathrm{t}$} & \multirow{3}{*}{ df } & \multirow{3}{*}{$\begin{array}{l}\text { Sig. } \\
(2 \text { colas })\end{array}$} \\
\hline & \multirow[t]{2}{*}{ Media } & \multirow[t]{2}{*}{$\begin{array}{l}\text { Dev. } \\
\text { Std. }\end{array}$} & \multirow{2}{*}{$\begin{array}{l}\text { Media } \\
\text { de } \\
\text { Error } \\
\text { Std. }\end{array}$} & \multicolumn{2}{|c|}{$\begin{array}{lr}\text { Intervalo con } \\
95 \% \\
\text { confidencia }\end{array}$} & & & \\
\hline & & & & Bajo & Alto & & & \\
\hline $\begin{array}{l}\text { Potencial Horizontal } \\
\text { Izquierdo-Derecho }\end{array}$ & 22.00 & 217 & 69 & -133 & 177 & 0.321 & 9 & 0.756 \\
\hline $\begin{array}{l}\text { Potencial Horizontal } \\
\text { Izquierdo-Vertical } \\
\text { Arriba }\end{array}$ & 84.00 & 205 & 65 & -63 & 230 & 1.294 & 9 & 0.228 \\
\hline $\begin{array}{l}\text { Potencial Horizontal } \\
\text { Izquierdo-Vertical } \\
\text { Abajo }\end{array}$ & - & 299 & 95 & -238 & 190 & $\begin{array}{l}- \\
0.254\end{array}$ & 9 & 0.805 \\
\hline $\begin{array}{l}\text { Potencial Horizontal } \\
\text { Derecho-Vertical } \\
\text { Arriba }\end{array}$ & 62.00 & 272 & 86 & -133 & 256 & 0.720 & 9 & 0.490 \\
\hline $\begin{array}{l}\text { Potencial Horizontal } \\
\text { Derecho-Vertical } \\
\text { Abajo }\end{array}$ & 46.00 & 159 & 51 & -160 & 68 & $\overline{-}-911$ & 9 & 0.386 \\
\hline
\end{tabular}

Nótese que, de los cinco sets comparados, sólo para el quinto par se obtiene una correlación estadística significativa entre el potencial horizontal derecho y el potencial vertical hacia abajo. La correlación se aprecia en la figura 5. Lo que implica que el potencial generado por el ojo para moverse a la derecha es mayor que el generado para moverse hacia abajo. Esto demuestra que el voltaje producido dado un desplazamiento ocular hacia arriba, a la izquierda y a la derecha es, estadísticamente, el mismo, mientras que si se compara el potencial generado en la exploración horizontal derecha v. el generado en la exploración vertical hacia abajo, el primero tiene una magnitud mayor. Este resultado se debe a la distancia máxima a la que puede desplazarse el ojo hacia abajo, la cual es más reducida que en las otras direcciones y 
concuerda con los resultados de campo visual para el ojo humano (Assefa et al., 2003; Marieb, 2008). Asimismo, queda validado el equipo diseñado.

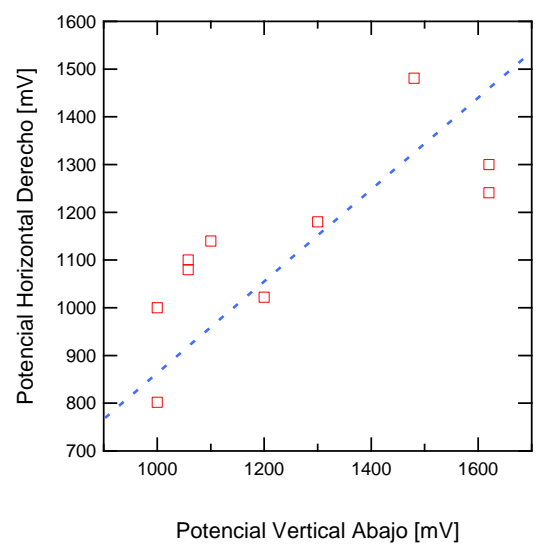

Figura 5: Prueba de diferencia de medias (prueba t)

\section{Limitaciones}

Existe una escasa información disponible en Panamá sobre investigaciones en oftalmología, motilidad ocular, y ortóptica. Así como también no se lleva una estadística sobre padecimientos visuales como estrabismo, ambliopía y la parecía típicamente tratadas mediante ortóptica. Ciertamente con un mayor entendimiento de la situación del discapacitado en el país, mejoraría la disponibilidad de dispositivos de alto desempeño para el diseño y fabricación de prototipos y la realización de estudios en el área.

\section{Conflicto de interés}

Los investigadores reportan que no existen conflictos de intereses como se declara en el ICMJE Form for Disclosure of Potential Conflicts of Interest.

\section{Financiamiento}

Esta investigación fue financiada en parte por la Universidad Especializada de las Américas a través del fondo de investigación No. 0052018.

\section{Conclusiones}

La investigación fue desarrollada para proveer una solución a un grupo particular de la población que necesita tecnologías asistidas para desempeñarse efectivamente en actividades ubicuas para el resto de la población.

El análisis estadístico de la investigación confiabiliza los resultados captados por el dispositivo diseñado (captador de señales mioeléctricas): 1.Se captaron, y trataron las señales, 2.Los resultados son similares a los de campo 
visual para el ojo humano lo que demuestra que el dispositivo funciona correctamente.

Al encender el led se muestra la posibilidad de que el dispositivo sea empleado para interfaz hombre-máquina.

\section{References:}

1. Assefa, N., \& Yosief, T. (2003). Lecture Notes for NUrsing Students: Human Anatomy and Physiology. Ethipioa Public Health Training Initiative.

2. Atique, M. M. U., Rakib, S. H., \& Siddique-e-Rabbani, K. (2016). An electrooculogram based control system. In 2016 5th International Conference on Informatics, Electronics and Vision (ICIEV) (pp. 809812). IEEE. https://doi.org/10.1109/ICIEV.2016.7760113

3. Bulling, A., Ward, J. A., Gellersen, H., \& Troster, G. (2011). Eye Movement Analysis for Activity Recognition Using Electrooculography. IEEE Transactions on Pattern Analysis and Machine Intelligence, 33(4), 741-753. https://doi.org/10.1109/TPAMI.2010.86

4. Bulling, A., Ward, J. A., Gellersen, H., \& Tröster, G. (2008). Robust Recognition of Reading Activity in Transit Using Wearable Electrooculography, 19-37. https://doi.org/10.1007/978-3-54079576-6_2

5. Dalgaard, N., González, E., \& Tulli, J. (2007 a). Escritura Mediante Señales Electrooculográficas. Nuevas Ideas En Informática Educativa, 3, 397-411.

6. Dalgaard, N. M., Luvoni, S., González, E. L., Tulli, J. C., Agüero, P. D., \& Uriz, J. (2007 b). Comunicador controlado por señales Electrooculográficas.

7. Heide, W., Koenig, E., Trillenberg, P., Kömpf, D., \& Zee, D. S. (1999). Electrooculography: technical standards and applications. Electroencephalography and Clinical Neurophysiology., 52, 223-240.

8. Jacob, R. (1993). Eye movement-based human-computer interaction techniques: Toward non-command interfaces. Advances in HumanComputer Interaction, 4, 151-190.

9. Jager, R. D., Mieler, W. F., \& Miller, J. W. (2008). Age-Related Macular Degeneration. New England Journal of Medicine, 358(24), 2606-2617. https://doi.org/10.1056/NEJMra0801537

10. Mahecha, D., Lozano, F., \& García, E. (2008). Control de mouse através de señales EOG y algoritmos de Boosting, 57-60.

11. Marieb, E. (2008). Anatomía y fisiología humanas (9th ed.). Pearson Addison Wesley. 
12. Martín, C. R. N., Correa, C. M., \& Schneider, O. (2006). Asimetría facial y estrabismo. Revista Mexicana de Oftalmologia, 80(1), 30-36.

13. Rodriguez, M. (2011). Captación de Señales Mioeléctricas Oculares. Universidad Especializada de.las Américas. Tesis de grado.

14. Salvucci, D. D., \& Anderson, J. R. (2001). Automated eye-movement protocol analysis. Human-Computer Interaction, 16(1), 39-86. https://doi.org/10.1207/S15327051HCI1601_2

15. Singh, T., Perry, C. M., \& Herter, T. M. (2016). A geometric method for computing ocular kinematics and classifying gaze events using monocular remote eye tracking in a robotic environment. Journal of NeuroEngineering and Rehabilitation, 13(1), 1-17. https://doi.org/10.1186/s12984-015-0107-4

16. Wade, N. J., \& Tatler, B. W. (2009). Did Javal measure eye movements during reading? Journal of Eye Movement Research, 2(5), 1-7. https://doi.org/10.16910/jemr.2.5.5

17. Wade, N. J., Tatler, B. W., \& Heller, D. (2003). Dodge-Ing the Issue: Dodge, Javal, Hering, and the Measurement of Saccades in EyeMovement Research. Perception, 32(7), 793-804. https://doi.org/10.1068/p3470

18. Wick, B., \& London, R. (1980). The Hirschberg test: analysis from birth to age 5. Journal of the American Optometric Association, (December). Retrieved from http://ukpmc.ac.uk/abstract/MED/7440867

19. Zhan, Z., Zhang, L., Mei, H., \& Fong, P. S. W. (2016). Online learners' reading ability detection based on eye-tracking sensors. Sensors (Switzerland), 16(9). https://doi.org/10.3390/s16091457 